\title{
Influence of interface cables termination impedance on radiated emission measurement
}

\author{
M. Bittera, V. Smiesko \\ Department of Measurement, Faculty of Electrical Engineering and Information Technology, \\ Slovak University of Technology, Ilkovicova 3, 81219, Bratislava, Slovakia, mikulas.bittera@stuba.sk
}

\begin{abstract}
Interface cables and especially their arrangement and terminations significantly affect the results of the radiated emission measurement of tested equipment. Two types of shortened two-wire interface cables were surveyed to obtain the influence of cable's termination. Two analyses, based on theoretical knowledge, were performed - the analysis using numerical simulation and the measurement of input impedance of the cable using network analyzer. The methods of analysis were verified and compared with real radiated emission measurements. Finally, the influence of cable termination was obtained and discussed.
\end{abstract}

Keywords: radiated emission measurement, cable terminator, two-wire cable

\section{INTRODUCTION}

$\mathrm{R}$ adiated emission measurement is a very complex measurement due to many disturbing factors affecting the process and results of the measurement. During the radiated emission measurement we shall identify the maximum intensity of unwanted electromagnetic field radiated from the equipment under test (EUT), measure it and compare with limit values. Because of reproducibility, the measurement is performed strictly according to the international standard [1]. Also EUT shall be in typical mode of operation and a test arrangement of EUT and its auxiliary devices shall be representative of typical installation practice. Such arrangement requirements are necessary to ensure reproducibility and also repeatability of the radiated emission measurement. These requirements are so strict because the influences of equipment, which is necessary for performing EMI measurement, and of test site arrangement, can be included into measurement correction or its uncertainty, but the effect of the EUT cannot (the EUT is not the same in different measurements and therefore its effect is not analysed) [2].

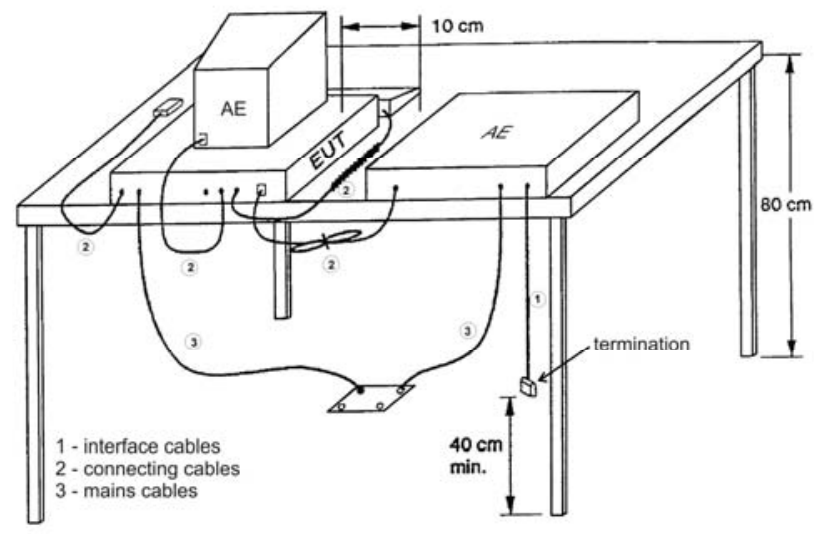

Fig.1. Test place arrangement of tabletop EUT for radiated emission measurement $(\mathrm{EUT}=$ equipment under test, $\mathrm{AE}=$ auxiliary equipment)
The most problematic part of EUT are its cables and theirs arrangement (see Fig.1) [2,3]. Interface cables shall be connected to each interface port of EUT, while the type and length of cables shall be specified by equipment manufacturer. The cables should be no longer than $0.4 \mathrm{~m}$. There is no obligation to terminate the interface cables that are not connected to other auxiliary equipment. Only if it is necessary for normal operation of EUT, the ends of such cables can be terminated by real impedance [1]. In the paper, we concentrate on the role of termination of a twowire cable attached to an electrically small tabletop EUT as a prospective radiator of disturbance. The cable may be shortened destructively or not - by arranging the cable into a bundle. Differential mode disturbance is applied on the analyzed cables and termination's effects on EMI measurement reproducibility are surveyed using various methods.

\section{SUBJECT \& METHODS}

\section{A. Theoretical analysis}

In terms of radiation, the behavior of the mentioned cable can be expressed using the transmission line method. For a transmission line, it can be shown that the input impedance $Z_{\text {in }}$ of a cable of length $l$ and loaded by an impedance $Z_{L}$ is [4]

$$
Z_{\text {in }}=Z_{0} \frac{Z_{L}+Z_{0} \tanh \gamma}{Z_{0}+Z_{L} \tanh \gamma}
$$

where $\gamma$ is the propagation constant and $Z_{0}$ is the characteristic impedance of the transmission line. Having the distance $D$ between the wires of the cable and the radius $d$ of the conductors, the characteristic impedance of the twowire cable in medium with permittivity $\varepsilon$ is given

$$
Z_{0}=\frac{120}{\sqrt{\varepsilon}} \cosh ^{-1}\left(\frac{D}{d}\right)
$$

while propagation constant $\gamma$ is also a function of resistance 
$R$, conductivity $G$, inductance $L$ and capacitance $C$ of the cable, which are dependent on material parameters of the cable and its surroundings

$$
\gamma=\sqrt{(R+j \omega L)(G+j \omega C)}
$$

Using substitution (2) into (1) it is possible to compute the current $I$ through the transmission line using Ohm's law. The using of transmission line theory was verified also by the real measurement with network analyzer on a selected model of the two-wire line.

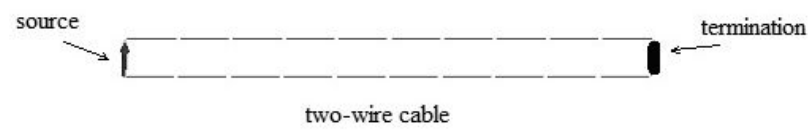

Fig.2. Two-wire cable as a rectangular loop antenna

No doubt that the two-wire cable behaves as an antenna (see Fig.2). To determine its radiation properties the wire can be represented by a rectangular loop antenna. In this case the magnitude of E-field component of electromagnetic field $E$ in arbitrary point of the surrounding space specified in spherical coordinate system $(r, \phi, \theta)$ is then given [5]:

$$
E=\frac{8 \eta I}{r} \frac{\sin \left(\frac{k a}{2} \sin \theta \cos \phi\right) \sin \left(\frac{k b}{2} \sin \theta \sin \phi\right)}{\sin \theta \sin 2 \phi}
$$

where $I$ is feed current flowing through the loop antenna, $\eta$ is free space wave impedance, $k$ is phase constant; $a$ and $b$ represent the dimensions of the rectangle. In our case, it is evident that $a » b$, so the transmission wire can be compared to a folded dipole. Hence, if the current is uniform along the antenna, the radiation is very weak for small $b$, since the radiation from the two long arms of the antenna is almost nullified. Of more interest would be the case when the current is not uniform (if $a \approx \lambda$ ), consequently the currents in the two long arms flow in the same direction [6]. While the shorter side of the loop $b$ « $\lambda$, we get the E-field radiated from the transmission line by simplification of equation (4):

$$
E=\frac{\eta k b I}{r} \frac{\sin \left(\frac{k a}{2} \sin \theta \cos \phi\right)}{\sin \phi}
$$

The equations (4) and (5) have two main disadvantages. They are suitable only for computing the far-field E-field, which is not fulfilled in case of lower frequencies of our interest and they cannot calculate with other surrounding material as free space does. Therefore it is necessary to find another method.

\section{B. Numerical simulation}

The [2], [3] and [7] show the advantage of numerical simulations that constitute a powerful tool to analyze such structures as wire cables. Nowadays, there are many electromagnetic field simulators used to solve electromagnetic problems. They are very useful, especially when the solved problem has no analytical solution. In our analysis of radiation of antennas and other wire structures, the most popular simulators are based on the method of moments.

Method of moments (MoM) solves Maxwell's equations in their integral form and in the frequency domain [8]. The principle of MoM is based on discretizing the solution domain, on dividing the analyzed structures to smaller parts, called segments. Then integral equation could be transformed to the system of linear equations, so the matrix is built, and then is inverted or it uses iteration to find the solution. The matrix building and its solution must be repeated for each frequency. In general, if the structures have more segments it means that one needs more long time calculations, but has more accurate results. So, it is necessary to strike a balance between the number of segments and calculation accuracy [7].

For our analysis the electromagnetic field simulator FEKO was used [9]. This simulator allows compute field distribution for different wire, surface and volume metallic structures. To get the appropriate results using the simulator, it is important to choose the proper model of analyzed structure. In the case of two-wire cable, the simplest model is represented by a rectangular antenna, as it was mentioned above; it consists of two parallel wires with signal source and termination. Also, a more complex system could be solved, so the numerical approach is used to survey the behavior of cables with bundles.

\section{Measurement}

Two types of measurements may be executed to analyse the influence of cable termination. First, the measurement of radiated emission, according to [1], was executed. The Efield radiated by the cable was measured in a semi-anechoic chamber, according to Fig.3, by measuring the antenna in $3 \mathrm{~m}$ distance. The antenna's height over the ground plane was $1 \mathrm{~m}$. The measured cable was hanging down, the highest point of the cable - the source - was in $0.8 \mathrm{~m}$ height and the lowest point - the termination - in $0.4 \mathrm{~m}$ height over the ground plane. Such measurement, by itself, represents the most objective analysis in spite of the fact that its results are loaded with measurement errors. But these errors are generally the same ones that influence the measurement of radiated emission [10] and therefore in this case their effect can be neglected. On the other hand, it is not possible to survey very simple structures due to the impossibility of their realization.

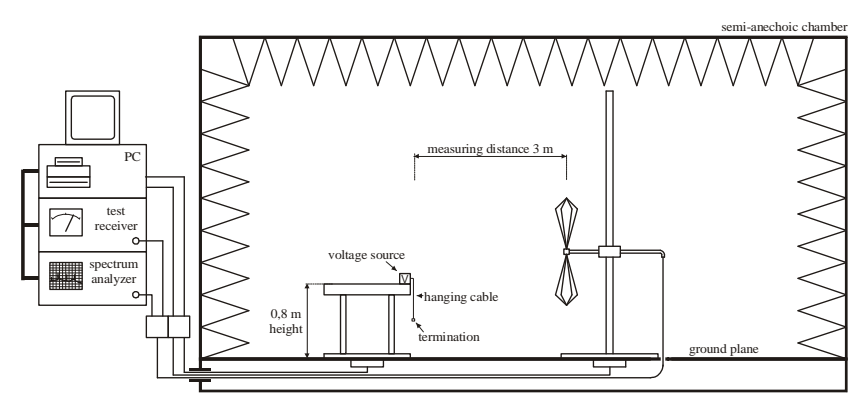

Fig.3. The measurement of E-field from the cable 
The other measurement is based on measuring of the input impedance - parameter S11 - of the cable by a network analyzer. According to previous theoretical analysis, the radiation from cables is dependent on the input impedance of the interface cable.

\section{RESULTS}

First, a simple $40 \mathrm{~cm}$ long two-wire cable was chosen as the model of the interface cable, which consists of two parallel wires with $50 \Omega$ termination on one end and with point voltage source on the other end. The diameter of these wires is $0.4 \mathrm{~mm}$ and the distance between them is $1.8 \mathrm{~mm}$. The long two wire cables are coated in a plastic insulator. The input impedance $Z_{\text {in }}$ of this model was obtained using all the mentioned methods. In Fig. 4 one can see the conformity of the results. Even though the first impedance maximum is expected at first resonance frequency about $187 \mathrm{MHz}$, the permittivity of the isolating material, which is higher than permittivity of free space, causes the shift of resonance frequency to the lower values (about $110 \mathrm{MHz}$ ). Also values of supply current $I$ and electric field $E$ in frequency range $30 \div 300 \mathrm{MHz}$ obtained by theoretical computation using (1) and (4) and numerical simulation of the selected model, but without insulation, are shown in Fig.5. We assume that signal voltage has the constant level of $1 \mathrm{~V}$ in the whole frequency range. In case of current comparison, their frequency dependences have the same tendencies; only the frequency of maximum calculated current is slightly moved to higher values of frequencies. On the other hand some differences of E-field values in distance $3 \mathrm{~m}$ are evident especially at lower frequencies. It is because in equation (5) we consider only the far-field components of E-field. The validation of this model using real measurement can be found in [3].

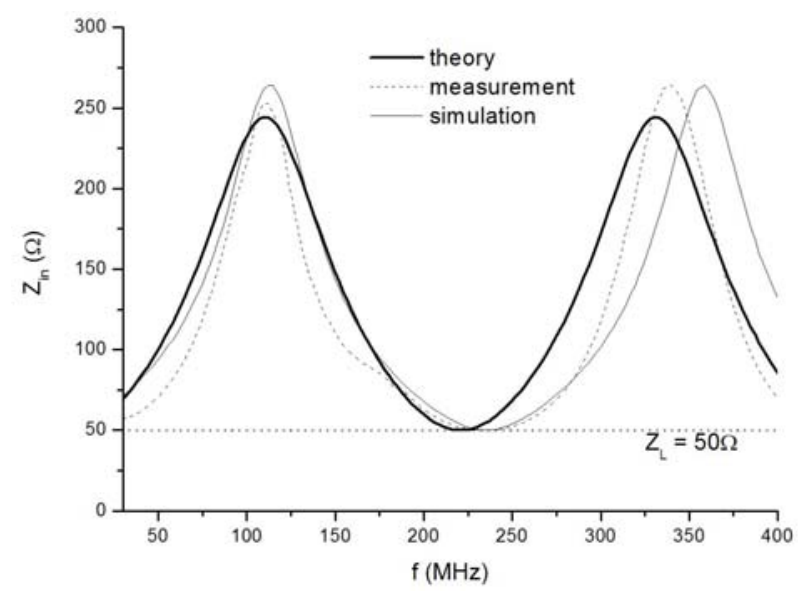

Fig.4. Frequency dependence of input impedance of $40 \mathrm{~cm}$ twowire cable for $50 \Omega$ load

It is expected that cable terminated by load impedance $Z_{L}$, which is equal to characteristic impedance $Z_{0}$, has the lowest unwanted radiation. In most cases, unfortunately, we do not know the impedance $Z_{0}$. Then the determining parameter is input impedance $Z_{\text {in }}$ of the analyzed cable. It can be obtained simply by measurement using the network analyzer (see Fig.6). The input impedance $Z_{\text {in }}$ determines the behavior of such cables; we usually expect the maximum of radiation if $Z_{i n}$ is in its minimum and vice-versa.

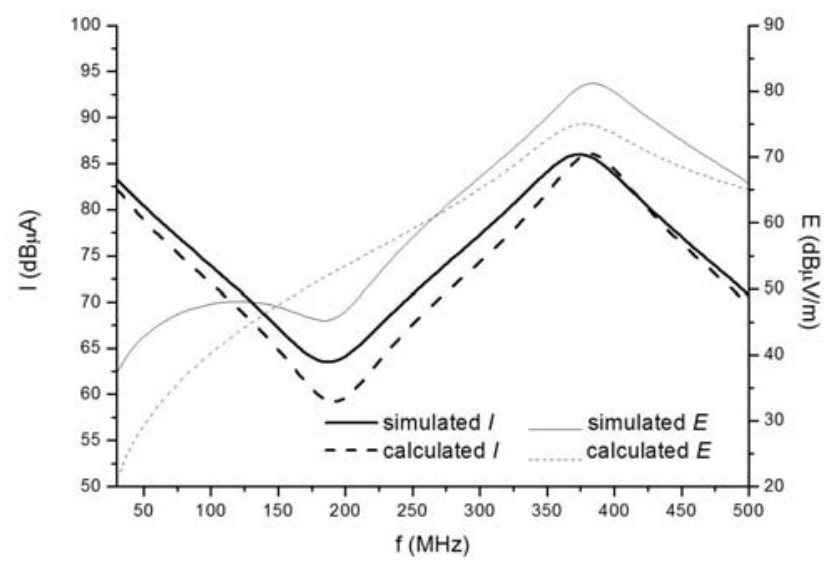

Fig.5. Frequency dependence of supply current of $40 \mathrm{~cm}$ two-wire cable and radiated field in $3 \mathrm{~m}$ distance

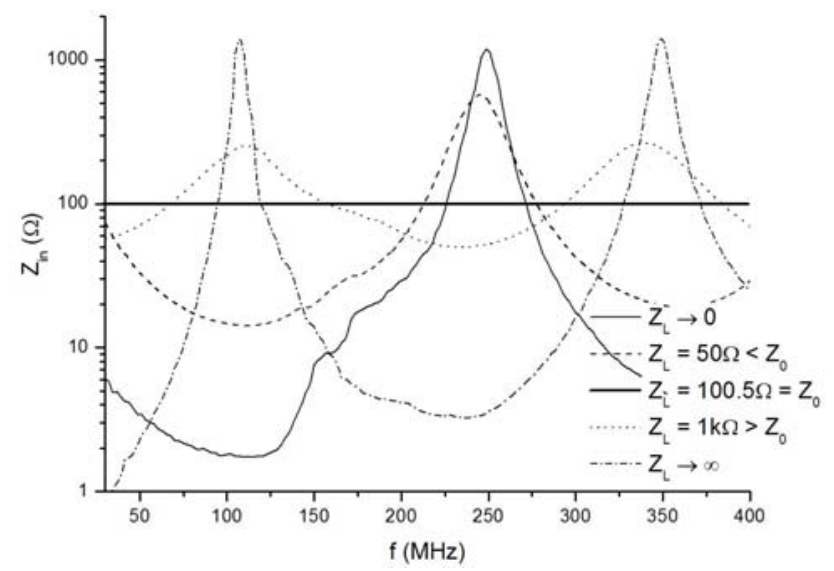

Fig.6. Measured values of input impedance $Z_{\text {in }}$ of $40 \mathrm{~cm}$ two-wire cable for different terminations $Z_{L}$

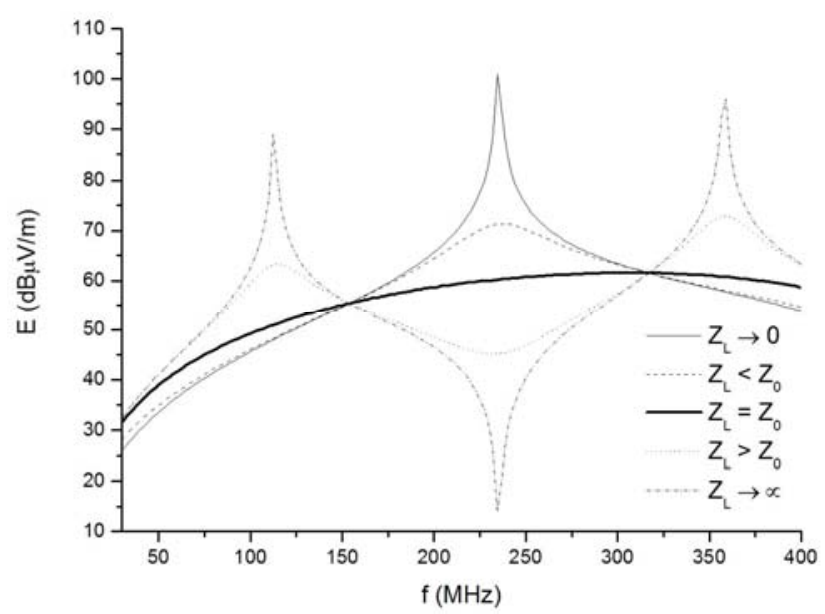

Fig.7. Frequency dependence of radiated E-field from $40 \mathrm{~cm}$ twowire cable as a function of terminator value $Z_{L}$ in free space

To confirm our expectation we used a numerical simulator with a selected model - simply hanging $40 \mathrm{~cm}$ long two-wire cable with given parameters. Two different 
simulations were performed - to calculate E-field in $3 \mathrm{~m}$ distance from the cable in free space and in presence of ground plane of the test place. The results of simulations are shown in Figures 7 and 8.

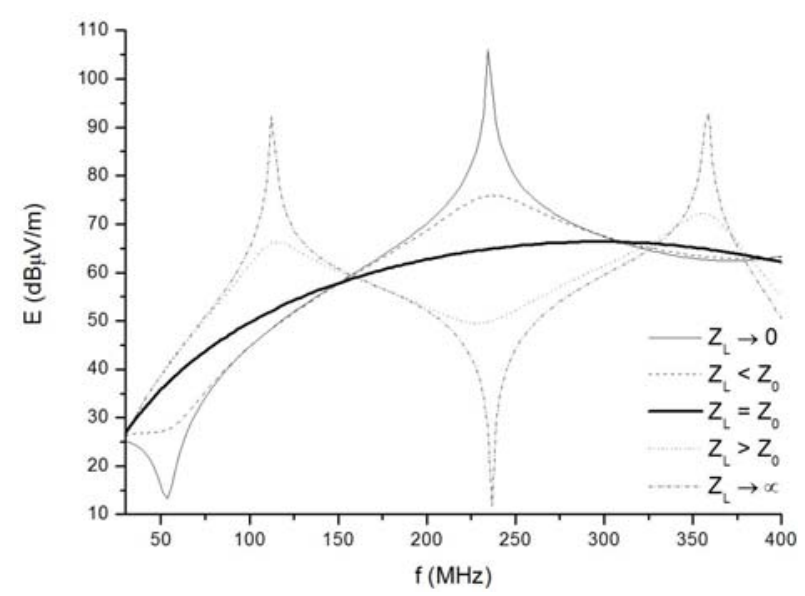

Fig.8. Frequency dependence of radiated E-field from $40 \mathrm{~cm}$ twowire cable as a function of terminator value $Z_{L}$ with the presence of ground plane

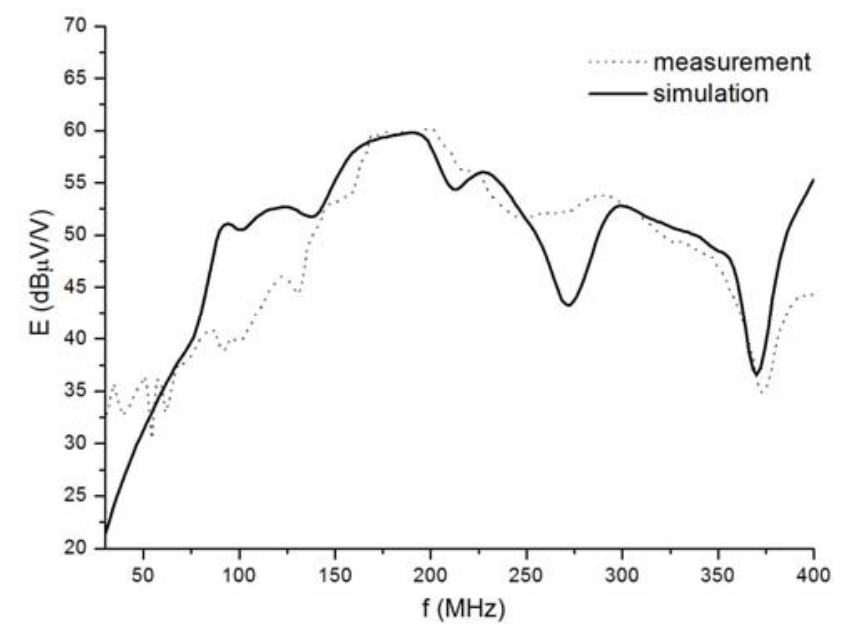

Fig.9. Comparison of measured and calculated value of E field of $40 \mathrm{~cm}$ two-wire cable for $50 \Omega$ load

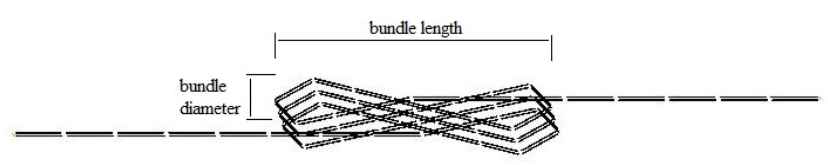

Fig.10. Model of two-wire bundled cable

The using of numerical simulations is justified also by a comparison of obtained values by simulations and the measurement of emission in test place (see Fig.9). Because it is not possible to create an ideal point voltage source, another model was created based on a real source. This source of wideband disturbance was situated in a metallic box, which is necessary due to undesired disturbance suppression, with dimensions $10 \times 10 \times 10 \mathrm{~cm}$, a two-wire cable was connected to the source at the middle of a square side. All the measurements were performed in semi- anechoic chamber of STU FEI, to avoid disturbance from surroundings, at $3 \mathrm{~m}$ distance. These results were also affected by reflection from the reference ground plane, which was in the chamber with and by the receiving antenna.

In the next analysis, another more complicated model, consisting of voltage source in a metallic box and attached wire, was used. The behavior of $1 \mathrm{~m}$ long two-wire cable folded at the cable center into a bundle was surveyed. There are some degrees of freedom, which can be changed to arrange the longer cables to be not longer than $0.4 \mathrm{~m}$. For our purposes the bundle was created as meanders (not loops) and joined in its center. The basic considered configuration was chosen as $1 \mathrm{~m}$ long two-wire cable folded in the center into a $14 \mathrm{~cm}$ long bundle with two meanders and $3 \mathrm{~cm}$ diameter of bending. Then the maximum length of such a cable is exactly $40 \mathrm{~cm}$. The whole analysis was performed for vertically polarized waves, where higher radiation was supposed.

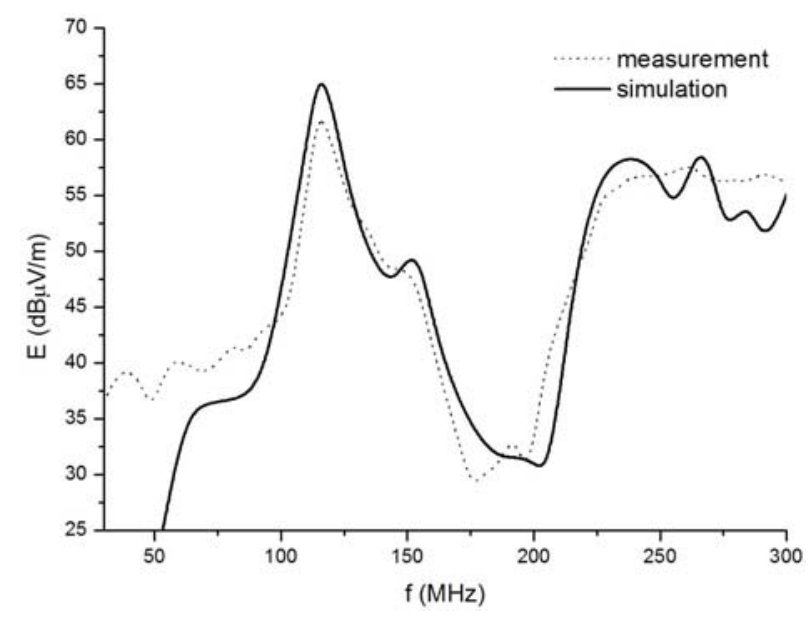

Fig.11. Frequency dependence of radiated E-field from bundled two-wire cable in $3 \mathrm{~m}$ distance.

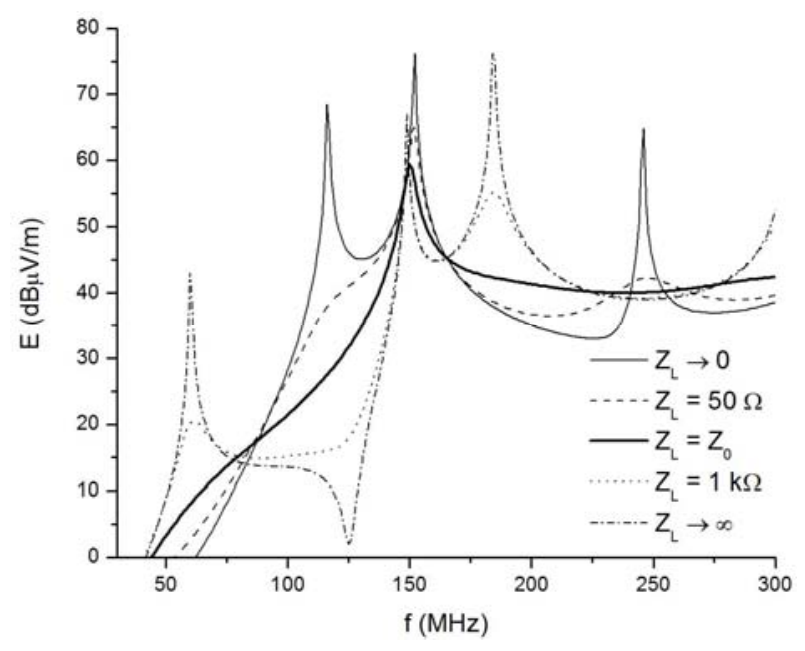

Fig.12. Frequency dependence of radiated E-field from bundled two-wire cable as a function of terminator value $Z_{L}$ with ground plane

The radiated emission measurement of such a model was performed. The results of measurements were influenced by 
all the equipment present in the test place and they were loaded by uncertainty of the entire measuring chain (similarly to the case of the previous model). Also simulation based on this real model was performed. The reference ground and receiving antenna, in height of $1 \mathrm{~m}$ above the ground and in $3 \mathrm{~m}$ distance from the cable, were included into the simulation model to get the most realistic results of analysis. As it can be seen in Fig.11, there are only small differences between measured and simulated values of the E-field in test site at point of the receiving antenna. It is important to notice that frequencies of maximum E-field values are the same. The difference at frequencies lower than $100 \mathrm{MHz}$ is caused just by high noise level of measuring equipment.

In Fig.12 the influence of E-field radiation of the cable on termination value is shown. The $1 \mathrm{~m}$ cable was considered, which was folded into the bundle, and was supplied by using point voltage source. As it can be seen, more frequency maximums of radiation can be detected.

\section{DISCUSSION}

As we can see from the obtained dependences, in the case of simple cable the E-field from a cable terminated by $Z_{0}$ is not constant but without evident or sharp extremes. Using terminators with lower values of $Z_{L}$ the cable behaves as a folded dipole and for higher values of $Z_{L}$ as a dipole with half value of maximum radiation frequency. The level of radiated E-field depends on the difference between values of terminating impedance $Z_{L}$ and characteristic impedance $Z_{0}$ of the cable. So, as expected, the worst results of EMI measurement were obtained when the interface cable was used with open or short circuit. Note in Fig.4 that for lower values of $Z_{L}$ there are no minimums in the E-field frequency dependence, they appear just in case of ground plane (Fig.8). Otherwise, the ground plane presence does not influence the frequency dependences of-E field very evidently. Results shown in Fig.8 can help us find the frequency with maximum radiation.

In case of bundled cables, the effect of terminating impedance $Z_{L}$ is also significant as in the case of simple short cable (see Fig.12). In comparison to simple two-wire cable, more maximums of radiation were obtained. Instead of one radiation maximum at $235 \mathrm{MHz}$, there were three local maximums. The frequency of previous maximum is moved to lower frequency of $155 \mathrm{MHz}$. On the other hand, values of all the maximums are lower by approximately $20 \mathrm{~dB}$. So, there is a higher probability that EUT with bundled cable passes the EMI measurement test than in case of simple two-wire cable, if the cable is not terminated with its characteristic impedance $Z_{0}$, because in EMI measurement the radiated disturbing level is the decisive one.

\section{CONCLUSION}

Two types of shortened two-wire interface cable were presented, verified and analyzed to survey the properties and behavior in terms of potential radiation due to differential mode disturbance. The main aim of this analysis was to help designers to reduce unwanted radiation and to test engineers to find the EUT arrangement with maximum of the radiation, if the termination impedance is not uniquely determined by manufacturers or designers.

In general, it is no doubt that the character of cable radiation is given by the properties of the cable. The wrong termination can cause additional maximums of radiation and the consequent fail of the EMI measurement test. To get the frequencies of potential radiation maximums quickly one can also use the mentioned method - measurement of input impedance of the cable. In general, due to different cable arrangements it can shift especially the frequency of maximum radiation of interface cables while using proper terminating impedance and help us to suppress the level of their radiation.

\section{ACKNOWLEDGMENT}

This research was financially supported by the project VEGA 1/0551/09 and by the Ministry of Education of the Slovak Republic under grant 2003 SP200280202.

\section{REFERENCES}

[1] IEC Standard. (2008). Information Technique Equipment - Radio Disturbance Characteristics Limit and Methods of Measurement. CISPR 22. Edition 6.0.

[2] Bittera, M., Smiesko, V., Kovac, K. (2006). Problem of bundled two-wire cable of tested equipment in emission measurement. Radioengineering, 15 (4), 22-26.

[3] Bittera, M., Smiesko, V., Kovac, K. (2007). Interference cable effect to EMI measurement results. In Meditarreanen Microwave Symposium, 14-16 May 2007. Budapest, Hungary, 159-162.

[4] Christopoulos, C. (2006). The Transmission-Line Modeling Method in Electromagnetics. 1st ed. San Rafael, USA: Morgan-Claypool Publishers.

[5] Balanis, C.A. (2005). Antenna Theory - Analysis and Design. 2nd ed. New Jersey, USA: John Wiley \& Sons.

[6] McDonald, K.T. (2003, May). A parallelogram loop antenna. Retrieved September 28, 2006, from http://puhep1.princeton.edu/〜mcdonald/examples/loop antenna.pdf

[7] Hartansky, R., Hallon, J. (2009). Measurement of electric field sensor based on resistively loaded dipole. Vestnik iževskogo gosudarstvennogo techničeskogo universiteta, 43 (3), 123-126.

[8] Harrington, R.F. (1993). Field Computation by Moment Method. 1st ed. New Jersey, USA: IEEE Press.

[9] EM Software \& Systems-S.A. (2005, December). FEKO - User's Manual - Suite 5.1. Retrieved October 1, 2009, from http://www2.ifh.ee.ethz.ch/ fieldcom/pps-antenna/ doc/UserManual.pdf

[10] Bittera, M., Hallon, J., Kovac, K., Szolik, I. (2004). Some factors influencing uncertainty of radiated emission measurement. In XIV. International Conference on Electromagnetic Disturbances - EMD 2004, 22-24 September 2004. Vilnius, Lithuania, 161-164.

Received April 10, 2010, accepted December 18, 2010. 